\title{
Polaron and Bipolaron Defects in a Charge Density Wave: a Model for Lightly Doped $\mathrm{BaBiO}_{3}$
}

\author{
Ilka B. Bischofst, Vladimir N. Kosturi, Philip B. Allen \\ Department of Physics and Astronomy, State University of New York, Stony Brook, New York 11794-3800
}

(October 23, 2018)

$\mathrm{BaBiO}_{3}$ is a prototype "charge ordering system" forming interpenetrating sublattices with nominal valence $\mathrm{Bi}^{3+}$ and $\mathrm{Bi}^{5+}$. It can also be regarded as a three-dimensional version of a Peierls insulator, the insulating gap being a consequence of an ordered distortion of oxygen atoms. When holes are added to $\mathrm{BaBiO}_{3}$ by doping, it remains insulating until a very large hole concentration is reached, at which point it becomes superconducting. The mechanism for insulating behavior of more lightly-doped samples is formation of small polarons or bipolarons. These are self-organized point defects in the Peierls order parameter, which trap carriers in bound states inside the Peierls gap. We calculate properties of the polarons and bipolarons using the Rice-Sneddon model. Bipolarons are the stable defect; the missing pair of electrons come from an empty midgap state built from the lower Peierls band. Each bipolaron distortion also pulls down six localized states below the bottom of the unoccupied upper Peierls band. The activation energy for bipolaron hopping is estimated.

71.38+i, 71.45.Lr

\section{THE MODEL}

Pure $\mathrm{BaBiO}_{3}$ has a distorted perovskite structure [1]. The nominal valence of $\mathrm{Bi}$ is $4+$, leaving a single $s$ electron per $\mathrm{Bi}$ atom. If $\mathrm{BaBiO}_{3}$ had perfect cubic perovskite symmetry, then (in independent electron picture, or band theory) it would be metallic, with the Fermi level half into a broad band made of Bi $6 s$ electrons (partially antibonded with $\mathrm{O} 2 p_{\sigma}$ states.) The large size of the $\mathrm{Bi}$ $6 s$ orbital indicates that single-electron (band) approximation should be good [2]. Therefore the origin of insulating behavior should lie in the structural distortions which double the unit cell. The simplest interpretation is that $\mathrm{BaBiO}_{3}$ is a prototype $\mathrm{d}=3$ Peierls insulator with a simple lattice dimerization.

$\mathrm{BaBiO}_{3}$ can be hole-doped by $\mathrm{Pb}$ substitution for $\mathrm{Bi}$ or $\mathrm{K}$ substitution for $\mathrm{Ba}$. At a quite high critical concentration $x_{c}$ of holes per cell $\left(x_{c} \approx 0.65\right.$ for $\mathrm{Pb}$ or $\approx 0.35$ for $\mathrm{K}$ ), an insulator to metal transition occurs, with the metal a superconductor. The high superconducting transition temperature $\left(T_{c} \leq 30 \mathrm{~K}\right)$ is compatible with a conventional electron-phonon mechanism, but neither theory [3] nor spectroscopy [4] is able to make a convincing confirmation. Electron-phonon effects certainly play an important role in the insulating part of the phase diagram.
This paper explores the self-trapped polarons which occur at low doping and which provide the most plausible mechanism explaining how these materials remain insulating to such high hole concentrations $x_{c}$.

We use a simple and yet fairly realistic model [5] which gives a microscopic description of the Peierls distortion. The model is illustrated in Fig. 11. There is one electronic degree of freedom per $\mathrm{Bi}$ atom, the amplitude of the $6 s$ electron orbital at that site, with corresponding creation operator $c_{\ell}^{\dagger}$. The index $\ell$ is a composite, standing for $(\vec{\ell}, \sigma)$ where the integer vector $\vec{\ell}=\left(\ell_{x}, \ell_{y}, \ell_{z}\right)$ locates Bi atoms on a cubic lattice of lattice constant $a=4.28 \AA$, and $\sigma= \pm 1$ is the spin index. The Bragg vector of the dimerized lattice is $\vec{Q}=(\pi, \pi, \pi)$. The phase factor $\exp (i \vec{Q} \cdot \vec{\ell})=(-1)^{L}$, where $L=\ell_{x}+\ell_{y}+\ell_{z}$, separates the simple cubic lattice into two sublattices, $A$ and $B$, with $(-1)^{L}$ equal to 1 on $A$ and -1 on $B$. The 6 nearest neighbors of each atom lie on the opposite sublattice.

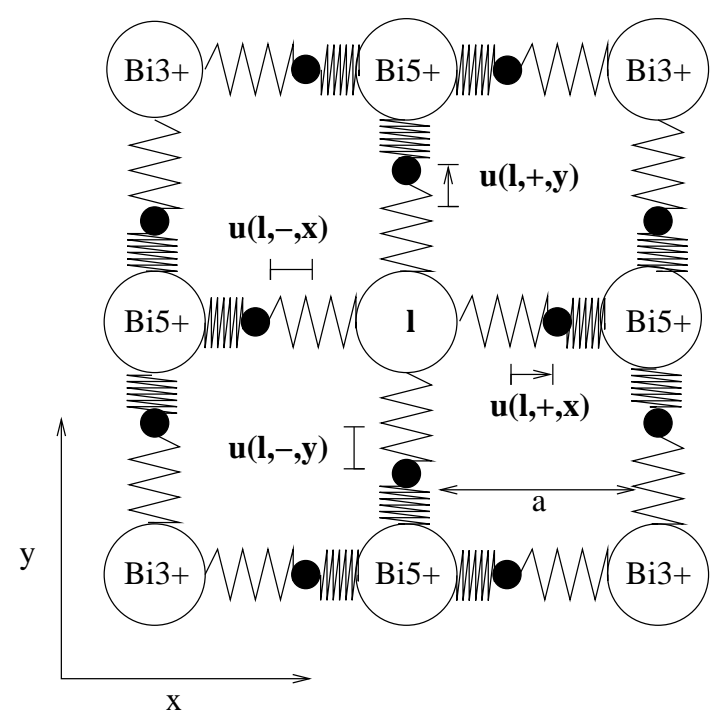

FIG. 1. Two-dimensional section of the Rice-Sneddon model. The small filled circles are oxygen atoms which are displaced by amounts $u$ from the midpoints of the bonds. The central $\mathrm{Bi}$ atom (labeled $\vec{l}$ ) is on the A sublattice (nominally $\mathrm{Bi}^{3+}$ ) where electrons accumulate because oxygens have moved away, lowering the potential energy of electrons.

In each cell there are three vibrational degrees of freedom, the oxygen displacements along the $\mathrm{Bi}-\mathrm{O}$ bond directions. Vibrations perpendicular to bond directions are assumed not to influence electrons. The oxygen displace- 
ments $u(\vec{\ell}, \alpha)$ are labelled by indices $\alpha=x, y, z$ which refer to the oxygen located at position $a \vec{\ell}+(a / 2) \hat{\alpha}$. It is convenient to define a local dilation or breathing amplitude $e(\vec{\ell})$ on the $\vec{\ell}$-th $\mathrm{Bi}$ atom,

$$
e(\vec{\ell})=\sum_{\alpha=x, y, z}[u(\vec{\ell},+, \alpha)-u(\vec{\ell},-, \alpha)]
$$

where $u(\vec{\ell}, \pm, \alpha)$ is the displacement of the oxygen atom at $a \vec{\ell} \pm(a / 2) \hat{\alpha}$. Thus $u(\vec{\ell},+, \alpha)$ is the same as $u(\vec{\ell}, \alpha)$, while $u(\vec{\ell},-, \alpha)$ is the same as $u(\vec{\ell}-\hat{\alpha}, \alpha)$, that is, it is the displacement of the oxygen atom located at $-(a / 2) \hat{\alpha}$ relative to the $\mathrm{Bi}$ atom $\vec{\ell}$. For a positive distortion $e(\vec{\ell})$, the Bi $6 s$ electron has its energy lowered by $-g e(\vec{\ell})$ where $g$ is the deformation potential. Each oxygen displacement costs elastic energy $(K / 2) u(\vec{\ell}, \alpha)^{2}$. Using the Raman measurement of $\hbar \omega=70 \mathrm{meV}$ for the oxygen "breathing mode" [6], the value of $K$ is $19 \mathrm{eV} / \AA^{2}$. The resulting Hamiltonian is

$$
\mathcal{H}=-t \sum_{<\ell, \ell^{\prime}>} c_{\ell}^{\dagger} c_{\ell^{\prime}}-g \sum_{\ell} e(\vec{\ell}) c_{\ell}^{\dagger} c_{\ell}+\frac{1}{2} K \sum_{\vec{\ell}, \alpha} u(\vec{\ell}, \alpha)^{2}
$$

where the hopping summation index $<\ell, \ell^{\prime}>$ goes over each nearest neighbor pair both forward and backward, and conserves spin. The variables $e(\vec{\ell})$ for different $\vec{\ell}$ are not independent, only the original variables $u(\vec{\ell}, \pm, \alpha)$ of Eq. (11) are independent. Piekarz and Konior [7] have introduced new decoupled breathing mode variables, at the expense of more distant terms in the coupling (second term) of Eq. (2).

For the time being we make the adiabatic approximation, treating oxygen mass $M$ as infinite (neglecting oxygen kinetic energy.) The electronic band structure is modelled with a simple $(s s \sigma)$ hopping integral $t$ between nearest neighbor $\mathrm{Bi}$ atoms. When there is no oxygen displacement, this yields the dispersion

$$
\epsilon(\vec{k})=-2 t\left(\cos \left(k_{x}\right)+\cos \left(k_{y}\right)+\cos \left(k_{z}\right)\right)
$$

Comparison with band theory 3,8 11] indicates that the value $t=0.35 \pm 0.5 \mathrm{eV}$ is appropiate for the band-width $12 t \approx 4 \mathrm{eV}$ of the conduction band in cubic $\mathrm{BaBiO}_{3}$. Our values for the parameters are summarized in table If.

TABLE I. Estimated values of parameters for $\mathrm{BaBiO}_{3}$. All parameters have $\approx 10 \%$ uncertainty.

\begin{tabular}{|ccc|}
\hline \hline \multicolumn{3}{c|}{ Fitted Parameters } \\
$t$ & $=$ & $0.35 \mathrm{eV}$ \\
$g$ & $=$ & $19 \mathrm{eV} / \AA^{2}$ \\
$g$ & Derived Parameters \\
$\Gamma=g^{2} / K t$ & $=$ & $1.39 \mathrm{eV} / \AA$ \\
$\delta=\hbar \omega / t$ & $=$ & 0.30 \\
\hline \hline
\end{tabular}

If energies are measured in units of $t$ and lattice displacements in units $\sqrt{t / K}$, then the model contains only a single dimensionless coupling constant $\sqrt{\Gamma}$ where $\Gamma=g^{2} / K t$. The dimensionless Hamiltonian $\mathcal{H} / t$ is just Eq.(2) with the substitutions $t \rightarrow 1, K \rightarrow 1$, and $g \rightarrow \sqrt{\Gamma}$. Later when non-adiabatic effects are treated, a new dimensionless energy ratio $\delta=\hbar \omega / t$ enters.

Because hopping goes only between inequivalent sublattices, the band energy has the symmetry property $\epsilon(\vec{k}+\vec{Q})=-\epsilon(\vec{k})$. The bands are symmetric around energy 0, which is the Fermi energy at half filling. There is perfect "nesting" at this value of the Fermi energy, because for every state with $\epsilon_{k}=0$, there is another state at wavevector $\vec{k}+\vec{Q}$ which is also at the Fermi energy. This perfect nesting property is an artifact of the nearest neighbor hopping, and is destroyed by nextneighbor hopping terms. Because of perfect nesting, it is particularly simple to find the ground state of the undoped (half-filled) case, obtaining a BCS-like integral equation for the Peierls gap $2 \Delta$, which has a solution $\Delta \approx 6.5 t \exp (-0.291 / \Gamma)$ for weak coupling $(\Gamma \ll 1)$. This was discussed in a previous paper [12]. The next section will treat this problem in the extreme atomic limit $(\Gamma \gg 1)$ where a particularly simple approximation $\Delta=12 \Gamma t$ applies.

An important consistency check on the Hamiltonian is the fact that three parameters $(t, K$, and $g$ ) fit four independently determined properties of $\mathrm{BaBiO}_{3}$. The value $g=1.39 \mathrm{eV} / \stackrel{\circ}{A}$ is chosen to yield a Peierls band gap $2 \Delta=$ $2 \mathrm{eV}$, agreeing with optical measurements [13. Then the oxygen sublattice displacement $u_{0}=\Delta / 6 g$ is predicted by the model to be $0.12 \AA$, while experiment sees $0.09 \AA$ 11, 14 .

Our model Hamiltonian contains a good explanation of the difficulty of making free carriers: doped-in holes self-localize, making small polarons and bipolarons. In a future paper we will re-examine the case of heavy doping, previously treated by $\mathrm{Yu}$, Chen, and $\mathrm{Su}$ [15. Here we concentrate on dilute doping. We have previously 16 looked at the criteria for polaron formation in the case of dilute doping into an empty band (no Peierls distortion), as occurs in materials like $\mathrm{BaSnO}_{3}$. The critical coupling strength $\Gamma_{c}(P)$ for polaron formation was found to be 1.96, and $\Gamma_{c}(B)$ for bipolaron formation was found to be 0.99 . We have also studied the case of dilute doping into the half-filled band by an analytic variational approximation [12]. Critical coupling strengths were dramatically reduced, to $\Gamma_{c}(P)=0.18$ and $\Gamma_{c}(B)=0.15$. Here we provide detailed numerical pictures of the behavior of lightly doped $\mathrm{BaBiO}_{3}$, including predictions about the optical spectrum. In a companion paper [17] we examine the self-trapped exciton state which forms when undoped $\mathrm{BaBiO}_{3}$ is excited optically. 


\section{EXTREME ATOMIC LIMIT $\Gamma \gg 1$}

The value $\Gamma \sim 0.30$ is appropriate for $\mathrm{BaBiO}_{3}$. This is intermediate between weak and strong coupling. Beyond its applicability to $\mathrm{BaBiO}_{3}$, the model is intellectually interesting in its own right, and we can be curious how its behavior evolves with coupling constant $\Gamma$ over the whole range 0 to $\infty$. We now solve the problem in the extreme atomic limit $\Gamma \rightarrow \infty$ which just means that we ignore the hopping term $(t \rightarrow 0)$, simplifying the mathematics greatly. The results yield insight into the intermediate region $\Gamma \sim 0.30$.

\section{A. Bipolaron crystal}

When the Bi $s$ level is half-filled, the strong-coupling solution consists of putting two electrons on each Bi atom on the $A$ sublattice and no electrons on each Bi atom on the $B$ sublattice. This is the extreme atomic limit of a Peierls charge density wave. The paired electrons can be given various names: "bipolarons" or "negative $U$ centers." The mechanism stabilizing these objects is oxygen motion. Each oxygen has as nearest neighbors one $A$ and one $B$ sublattice Bi atom. Therefore it experiences a force away from the more negative $A$ site and toward the more positive $B$ site. The piece of the Hamiltonian (2) containing this force is

$$
-g u(\vec{\ell}, \alpha) \sum_{\sigma}\left(c_{\ell}^{\dagger} c_{\ell}-c_{\ell^{\prime}}^{\dagger} c_{\ell^{\prime}}\right)
$$

where $\overrightarrow{\ell^{\prime}}=\vec{\ell}+\hat{\alpha}$ is the other member of the pair of nearest $\mathrm{Bi}$ atoms. Let us define the Peierls order parameter (amplitude of charge density wave) $\rho_{0}$ by

$$
\sum_{\sigma}<c_{\ell}^{\dagger} c_{\ell}>=1+(-1)^{L} \rho_{0} .
$$

In the undistorted cubic structure, $\rho_{0}=0$. In strong coupling, $\rho_{0} \rightarrow 1$, that is, the $\mathrm{Bi}$ atoms have charge 2 on sublattice $A$ and 0 on sublattice $B$. The force is $2 g \rho_{0}$ on each oxygen, directed toward its nearest $B$-type Bi neighbor, counteracted by a harmonic restoring force $K u$, yielding an optimum displacement $u_{0}=2 g \rho_{0} / K$ or a "breathing amplitude" $e(\vec{\ell})=(-1)^{L} e_{0}$, where, the oxygen breathing order parameter $e_{0}$ is $12 g \rho_{0} / K$. These relations remain true for arbitrary coupling $\left(0 \leq \rho_{0} \leq 1\right)$. In strong coupling, site energies of $\mathrm{Bi} s$ orbitals are thus $-12 g^{2} / K$ on $A$ sites and $+12 g^{2} / K$ on $B$ sites. For hopping $t=0$, the lowest energy excitation is to move one electron from an $A$ site to a $B$ site. This costs energy $2 \Delta_{0}=24 g^{2} / K=24 \Gamma t$ which is the value of the Peierls gap. We shall use the notation $\Delta_{0}$ to denote the value of the gap in the atomic limit, and $\Delta$ for the value for the actual coupling strength under consideration. The limiting answer $2 \Delta_{0}=24 g^{2} / K$ can also be found from the integral equation (23) of ref. [12], by taking $\Delta \gg t$.
We get a measure of the value $\Gamma_{c}$ where the crossover occurs between strong and weak coupling by comparing total energies. In the atomic limit, this is $-6 g^{2} / K$ per atom $\left(-12 g^{2} / K\right.$ per electron of on-site orbital energy, but at a cost of $+6 g^{2} / K$ per atom of elastic energy.) In the opposite $(g=0)$ limit, the electrons gain on average $-2 t$ of delocalization energy by band formation in the undistorted crystal. These energies are equal at $\Gamma_{c}=$ $1 / 3$.

So far we are safe in the adiabatic approximation, since the vibrational energy $\hbar \omega$ is small compared with the Peierls gap in strong coupling. However, the true lowest energy excitation will cost less than $2 \Delta$ once we allow relaxation of the atoms. Thus the optical spectrum will have non-adiabatic Franck-Condon character, with minimum excitation energy corresponding to a "self-trapped exciton." The companion paper [17 pursues this topic. The rest of this paper is devoted to the case where one or two holes are doped into the half-filled band. We will consider both the ground state and the new excitations induced by the doping.
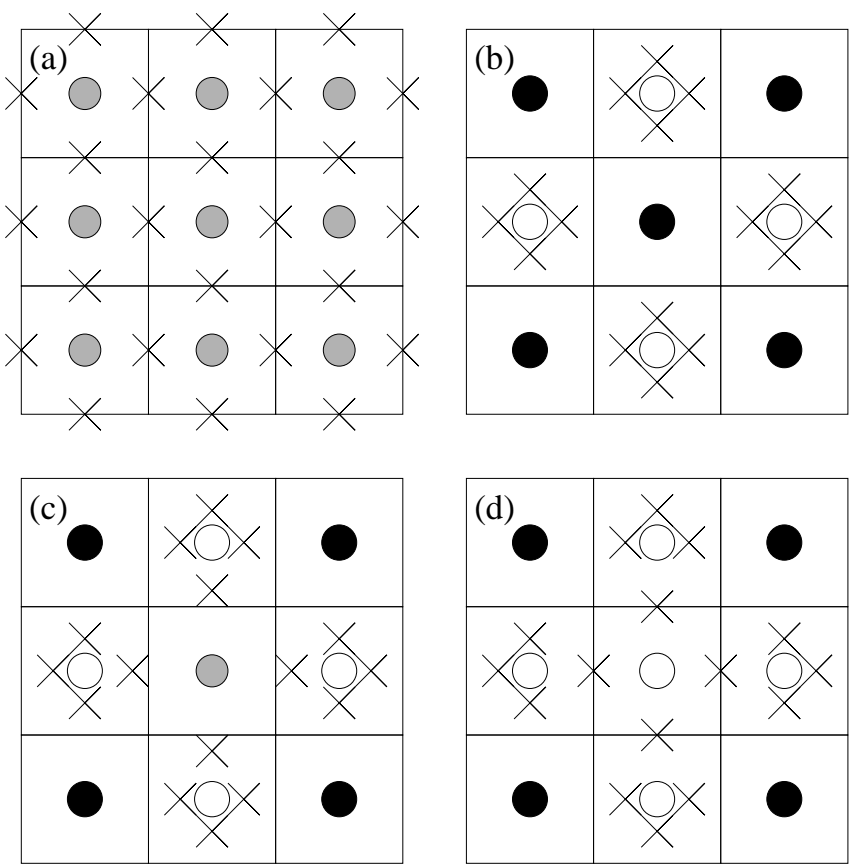

FIG. 2. Schematic structures: (a) is the undistorted high temperature structure; (b) is the low temperature Peierls phase of pure $\mathrm{BaBiO}_{3}$; (c) has a hole polaron in the central cell; and (d) has a hole bipolaron. Circles denote Bi atoms, with filled circles being $\mathrm{Bi}^{3+}$ ions with two $s$ electrons, shaded circles being $\mathrm{Bi}^{4+}$ ions with one $s$ electron, and open circles being $\mathrm{Bi}^{5+}$ ions with no $s$ electrons. X's denote oxygen ions.

\section{B. One hole in the atomic limit}

To remove an electron, keeping the Peierls distortion fixed, costs energy $12 g^{2} / K$ (the amount of Peierls attractive energy lost.) When $t=0$, the hole sits on 
some particular $A$ sublattice site as a singly charged $\mathrm{Bi}^{4+}$ ion. The 6 surrounding oxygens now experience a diminished repulsive force, $g$ instead of $2 g$, toward their $\mathrm{Bi}^{5+}$ neighbors. The local Peierls displacement $u_{1}$ is therefore $g / K$, or half of $u_{0}$. This reduces the elastic energy by 6 times $K\left(u_{0}^{2}-u_{1}^{2}\right) / 2$ or $9 g^{2} / K$ but does this at the expense of raising the energy of the $6 s$ orbital at this site from $-12 g^{2} / K$ to $-6 g^{2} / K$. Since this orbital is occupied once, the net energy saved by this local distortion is $3 g^{2} / K$. The resulting object, a point defect in the Peierls order parameter, is a small hole-polaron. In a system with $N$ Bi atoms, the isolated polaron corresponds to the system having $N-1$ electrons. Its energy $E[P] \equiv E_{\mathrm{gs}}(N-1)-E_{\mathrm{gs}}(N)$ is $9 g^{2} / K$, smaller than the removal energy $12 g^{2} / K$ when no relaxation is permitted. The difference is the polaron trapping energy, $E_{t}[P]=3 g^{2} / K$.

Photoemission would measure the polaron formation energy $9 g^{2} / K$ rather than $12 g^{2} / K$ provided the removal were done slowly enough that oxygen atoms could move to their new optimum positions. In fact, this is surely not the case, and one expects to see instead a peak photoemission intensity at the adiabatic energy $12 g^{2} / K$, with a series of Franck-Condon vibrational sidebands with a Gaussian envelope extending down to the threshold $9 g^{2} / K$. A detailed discussion of the corresponding effect in $\mathrm{LaMnO}_{3}$ was given earlier [18].

The hole polaron is shown schematically in part (c) of Fig. 2. It is clear that the orbitals on the surrounding 6 $\mathrm{Bi}^{5+}$ ions must also be altered by the oxygen relaxation. Rather than having their energy pushed up by $6 g u_{0}$, their energy is pushed up by $5 g u_{0}+g u_{1}$, or $11 g^{2} / K$ rather than $12 g^{2} / K$. Thus the polaron defect has an interesting spectrum of gap states. There is the singly occupied $A$ sublattice state located at $\Delta / 2$ above the lower Peierls band at $-\Delta$, and there are six empty $B$-sublattice states located at energy $\Delta / 12$ below the upper Peierls band at $\Delta$. This spectrum is shown on the left side of Fig. 3 .

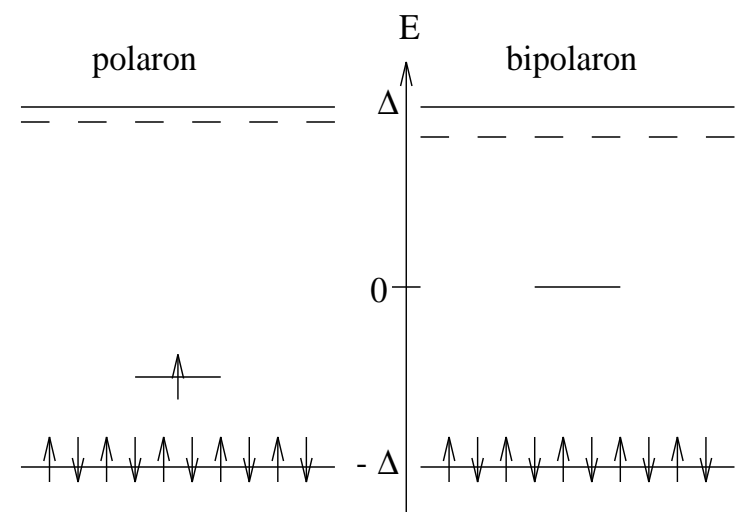

FIG. 3. Energy spectrum of the Rice-Sneddon model in the atomic limit $(t=0)$ with one hole present (left side labeled "polaron") or with two holes present (right side labeled "bipolaron.") In both cases, the valence band contains $N / 2-1$ doubly-filled states at energy $-\Delta$, and the conduction band has $N / 2-6$ degenerate states at energy $\Delta$, with 6 additional empty states bound below them.

\section{Two holes in the atomic limit}

Two polarons spatially separated cost two times $9 g^{2} / K$, but if they occupy the same local site (with opposite spin, $S=0$ ) then it is easy to see that the cost is only $12 g^{2} / K$. This is because removal of two electrons costs $24 g^{2} / K$ in lost Peierls energy, but when sitting on a single $A$ site (converting the $\mathrm{Bi}^{3+}$ to a $\mathrm{Bi}^{5+}$ ), the local strains on the six surrounding oxygens all disappear $\left(u_{2}=0.\right)$ This gains back $12 g^{2} / K$ that was previously paid in strain energy. The local $s$ orbital at the site of the bipolaron now has energy 0 because of no local strain, but unlike the polaron case, the loss of Peierls energy of this state does not alter anything since the level is now empty. Thus the total energy of the bipolaron is $E[B]=12 g^{2} / K$. Relative to free holes which cost $24 g^{2} / K$, the bipolaron trapping energy is $E_{t}[B]=12 g^{2} / K$. The energy released when two polarons bind into a singlet bipolaron is $E_{b}=6 g^{2} / K$. However, this neglects on-site Coulomb repulsion $U$. We expect a reduction of the bipolaron binding energy to $E_{b}=6 g^{2} / K-U$. The Hubbard $U$ is defined as the energy difference between charged ions $E\left(\mathrm{Bi}^{3+}\right)+E\left(\mathrm{Bi}^{5+}\right)$ and "neutrals" $2 E\left(\mathrm{Bi}^{4+}\right)$ for $\mathrm{BaBiO}_{3}$ with oxygen atoms frozen in cubic perovskite positions. Experimentally, the absence of free spins in doped $\mathrm{BaBiO}_{3}$ indicates that $\mathrm{Bi}^{4+}$ (with $S=1 / 2$ ) is disfavored. Therefore, $U$ cannot be large enough to destabilize bipolarons. Vielsack and Weber [2] have done constrained density functional calculations to obtain $U$. They fit their results to an effective $U$ of $0.6 \pm 0.4 \mathrm{eV}$ of a single band model like ours. This is similar in size to our attractive energy of $6 g^{2} / K \approx 0.6$ $\mathrm{eV}$. When hopping is turned back on, the attractive energy goes down, but the bipolaron also spreads out, so that the repulsive $U$ is less effective. Thus $U$ is small enough to permit bipolarons to exist.

The spectrum of $\mathrm{BaBiO}_{3}$ with a bipolaron consists of a filled lower Peierls band containing $N / 2-1$ states at energy $-\Delta=-12 g^{2} / K$, one empty midband state $E=0$, and $N / 2-6$ upper Peierls band states at energy $+\Delta$. Six new empty states appear below the empty upper Peierls band, at energy $5 \Delta / 6$. These are the $s$ orbitals on the six $B$ sites surrounding the bipolaron, which have only 5 out of 6 first neighbor oxygens with displacement $u_{0}$, and one undisplaced. The spectrum is shown on the right side of Fig. 3 . 


\section{D. corrections for small hopping $t$}

For the perfectly ordered half-filled band, the Peierls gap diminishes quadratically as $t=\Delta_{0} / 12 \Gamma$ is turned on,

$$
\Delta=\Delta_{0}\left(1-\frac{1}{48 \Gamma^{2}}+\cdots\right)
$$

This follows by expanding the integral equation (23) of ref. [12] for small values of $t / \Delta$. The amplitude $\rho$ of the CDW diminishes the same way, from 1 to $1-1 / 48 \Gamma^{2}+\cdots$.

The eigenstates are now extended Bloch states of energy $\lambda(k)= \pm \sqrt{\Delta^{2}+\epsilon(k)^{2}}$, where $\epsilon(k)$ is the eigenvalue of the undistorted lattice, $-2 t \sum_{\alpha} \cos \left(k_{\alpha}\right)$. Thus the least expensive delocalized hole states are at the top of the lower Peierls band where $\epsilon(k)=0$ and cost $\left(12 g^{2} / K\right)\left(1-1 / 48 \Gamma^{2}\right)$. The competing bipolaron state can no longer be completely localized on a site because the charge on the nominal $\mathrm{Bi}^{3+}$ ions is $1+\rho<2$. It is not easy to develop a systematic $1 / \Gamma$ expansion for the localized solutions. The problem is that as oxygens move and the polaron localizes, wavefunctions and charges of all occupied delocalized states must be altered self-consistently near the polaron.

\section{NUMERICAL STUDY OF POLARON}

The algorithms used are explained in detail in the MA thesis of the first author 19. A large supercell (typically between 100 and $1000 \mathrm{Bi}$ atoms) was chosen, with periodic boundary conditions applied. It was useful to have the translation vectors $\vec{A}, \vec{B}, \vec{C}$ of the supercell not lie along symmetry axes, but to use small asymmetrical deviations in order to lift degeneracies. The translation vectors all translate $A$ sublattice points to other $A$ sublattice points, so that the Peierls state fits the cell. Trial oxygen positions are chosen, the Hamiltonian matrix is diagonalized, and forces on oxygen atoms are calculated by summing over occupied wavefunctions to get the electronic charges on the two adjacent $\mathrm{Bi}$ atoms. A variable metric method [20] was used to iterate towards optimal oxygen displacements. We confirmed that for the half-filled case, the density of states, oxygen coordinates, and Peierls gap all agreed well with calculations by other methods. The half-filled ground state serves as the "vacuum" for the rest of our work. To study doping by one or two holes per supercell, a cell with $\approx 200$ atoms is sufficient if the coupling constant $\Gamma>0.22$. For smaller $\Gamma$, larger cells are needed.

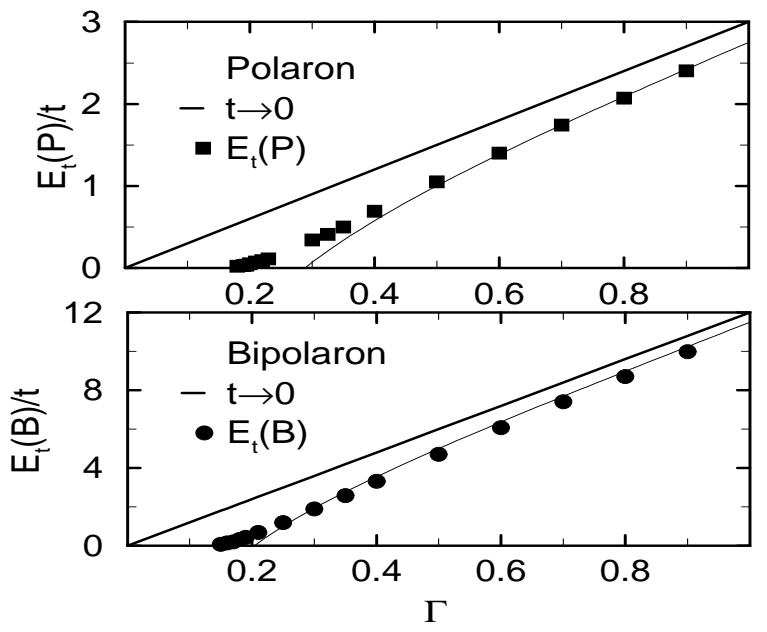

FIG. 4. Trapping energy of a polaron (upper panel) and a bipolaron (lower panel) computed numerically. Bold solid lines are strong-coupling predictions. Thin lines are approximate $1 / \Gamma^{2}$ corrections discussed in Sec. III.A.

\section{A. total energy}

By simply restricting the occupancy to be $N-1$ or $N-2$, we obtain solutions with polaron or bipolaron local defects. The numerical results for the "trapping energies" are shown in Fig. 4. Stable polaron solutions occur when $\Gamma>\Gamma_{c}(P) \approx 0.18$. Stable bipolaron solutions occur when $\Gamma>\Gamma_{c}(B) \approx 0.15$. At lower values of $\Gamma$ an inserted hole just stays at the top of the valence band, causing an order $1 / N$ renormalization of the Peierls gap. For $\Gamma>\Gamma_{c}$, such states were always found to be numerically unstable toward formation of local defects. The onset of localized solutions seems to occur continuously, unlike the case where an empty $s$-band is doped and localized solutions appear discontinuously (in adiabatic approximation) at much larger values $\Gamma_{c}=1.96$. There are several reasons for the ease of polaron formation in the Peierls state relative to the empty band. Perhaps foremost is the fact that there is less to be gained by delocalization, because lowenergy delocalized states are occupied. Also, the Peierls state has lattice strain already built in, so polaron formation can occur by reduction of the Peierls lattice strain rather than by initiation of new lattice strain.

Although we do not have a systematic $1 / \Gamma$ perturbation theory, nevertheless, a very simple correction to the $\Gamma \rightarrow \infty$ expression can be made. The trapping energy of polarons $E_{t}[P]$ is the difference between the energy $\Delta$ to create a hole without relaxation, and the energy $E[P]$ of the relaxed polaron. In the $\Gamma \rightarrow \infty$ limit, this gives $E_{t}[P] / t=12 \Gamma-9 \Gamma$. The first term, $\Delta_{0} / t$, the energy to put a hole into the "vacuum" without lattice relaxation, can be corrected by Eq. (6) to $\Delta(\Gamma) / t \approx 12 \Gamma-1 / 4 \Gamma$. We do not know the form of the correction to $E[P]$, but find that the correction is weaker than the vacuum correction. Therefore, correcting only the vacuum term gives the for- 
mula $E_{t}[P] / t \approx 3 \Gamma-1 / 4 \Gamma$. The $\Gamma \rightarrow \infty$ formula (labeled $t \rightarrow 0)$ and the vacuum-corrected formula are shown in Fig. 4 as thick and thin solid lines. The fit to the polaron is very good down to $\Gamma=0.5$. For the bipolaron, the corresponding formulas are $E_{t}[B] \equiv 2 \Delta-E[B]$, and $E_{t}[P] / t \approx 12 \Gamma-1 / 2 \Gamma$. The vacuum-corrected fit is not as good as for the polaron.

\section{B. size and shape of polarons}

In the $t \rightarrow 0$ limit, an inserted hole sits on a single site. Hopping causes the hole to spread, gaining delocalization energy. The amount of positive hole charge $\rho(\vec{r})$ at a site $\vec{r}$ is defined as

$$
\rho(\vec{r})=\sum_{i}^{\text {occ,vac }}\left|\Psi_{i, \operatorname{vac}}(\vec{r})\right|^{2}-\sum_{i}^{\text {occ, } \mathrm{P}}\left|\Psi_{i, P}(\vec{r})\right|^{2},
$$

where "vac" and $P$ indicate the single particle states $\Psi$ for vacuum and polaron cases. In Fig. 5, the hole charge at the central site $\vec{r}=0$ is plotted. For $\Gamma>0.5$, the polaron is almost completely on the central site, whereas for the bipolaron, the available charge on the central $A$ type atom is essentially all depleted for $\Gamma>0.4$, but until a somewhat larger $\Gamma$, some charge must be removed from surrounding sites. At the realistic value $\Gamma=0.3$, the polaron depletes 0.84 electrons from the central A site, and the bipolaron depletes 1.74 electrons, which is almost all of the 1.80 electrons which occupy A sites in the vacuum state.

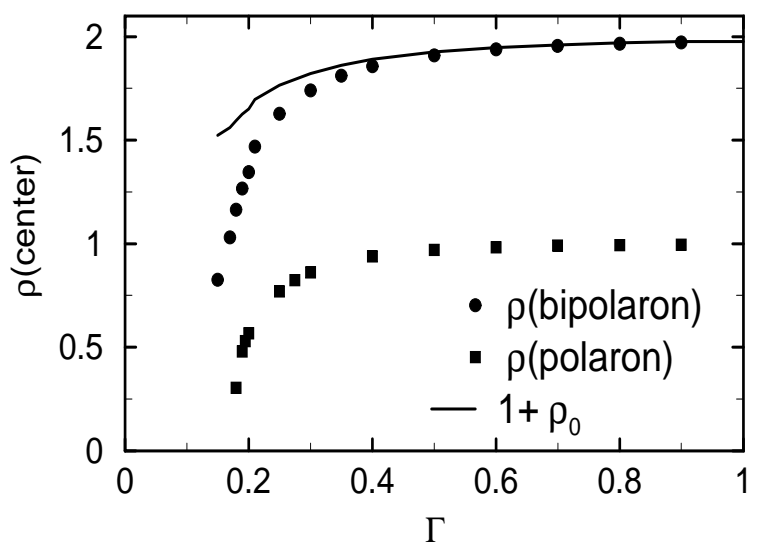

FIG. 5. The total charge removed from the central site of the hole polaron and bipolaron. The solid line is the total charge at that site in the Peierls state, the maximum available for removal.

The polaron defect diminishes the amplitude of the Peierls charge-density wave in its local vicinity. This is seen by examining the charge on atoms near the polaron. At $\Gamma=0.3$, to make a polaron, 0.16 electrons are taken from sites other than the central site. We find that neighboring B sites do not lose electron density, but rather gain electrons when a polaron is formed nearby; neighboring $\mathrm{A}$ sites are more depleted of electrons than if $\mathrm{B}$ sites had been unaffected. The distance-dependence of the hole charge is shown in Fig. (6) to be exponential, with decay length $0.39 a$. The corresponding decay length for the bipolaron disturbance is $0.32 a$.

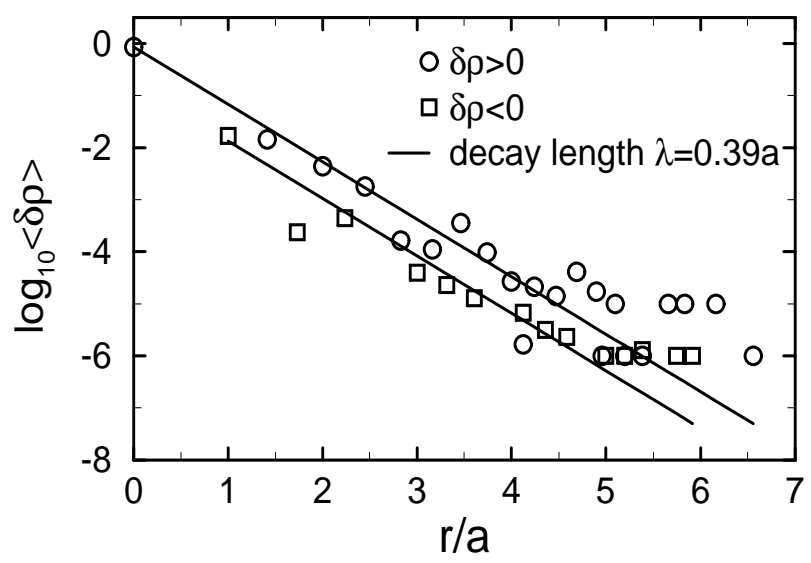

FIG. 6. Charge versus distance for the hole polaron when $\Gamma=0.30$. The calculation used a cell with $N=1000$. Sites with depletion of electron density ( $\operatorname{charge} \delta \rho>0$ ) are denoted by circles; sites with excess electron density $(\delta \rho<0)$ are denoted by squares. Beyond $r=5 a$, overlapping charge from neighboring cells affects the answer.

If small polaron formation occurs in continuous fashion for $\Gamma>\Gamma_{c}$, as suggested by Fig. 5 shows the continuous evolution with $\Gamma$, for $\Gamma>\Gamma_{c}$, from large CDW-like (bi)polarons to small (bi)polarons. The decay length $\lambda$ (defined as the negative reciprocal of $d \ln (\delta \rho) / d r$ ) should diverge as $\Gamma$ is reduced to $\Gamma_{c}$. This cannot be verified numerically because of the small linear size of available computational cells in 3 dimensions. We do see a trend in the right direction, shown in Table II.

TABLE II. Decay constants for the hole charges for a (bi)polaron

\begin{tabular}{|ccccc|}
\hline \hline$\Gamma$ & $\lambda_{p} / a$ & $\rho_{\text {center }}^{p}$ & $\lambda_{b} / a$ & $\rho_{\text {center }}^{b}$ \\
0.9 & 0.21 & 0.99 & 0.20 & 1.97 \\
0.6 & 0.24 & 0.98 & 0.23 & 1.94 \\
0.3 & 0.39 & 0.84 & 0.32 & 1.74 \\
0.2 & 0.57 & 0.58 & 0.41 & 1.40 \\
\hline \hline
\end{tabular}




\section{Conduction band edge states}

The polaron defect perturbs the band Peierls states in a local region. This perturbation is sufficiently strong to create new localized states at the bottom of the conduction band. This effect is particularly simple in the strong-coupling limit, where we already discussed (see Fig. 3 and nearby text) six states bound by $\Delta / 12$ (near a polaron) or $\Delta / 6$ (near a bipolaron). These states can be classified as one $s$-like $\left(A_{1 g}\right)$, a $d$-like doublet $\left(E_{g}\right)$ and a $p$-like triplet $\left(T_{1 u}\right)$. Therefore, as hopping $t$ is turned on, we can expect the six bound states to split into singlet, doublet, and triplet, and for weak coupling, perhaps to become unbound. The numerical results are shown in Fig. 7. The $s$-like state is bound only for $\Gamma>\Gamma_{c}(s) \approx$ 0.30 (for a polaron) or 0.28 (for a bipolaron), while the other states are nearly degenerate and look to persist all the way to the critical $\Gamma$ for polaron or bipolaron formation. Numerical finite-size effects prevent high accuracy in these estimates. The reason for the weaker binding of the $s$-like state is that it couples to the polaron state which splits off from the valence band, forming a spread out polaron with no radial node, and an empty state bound below the conduction band with a radial node.

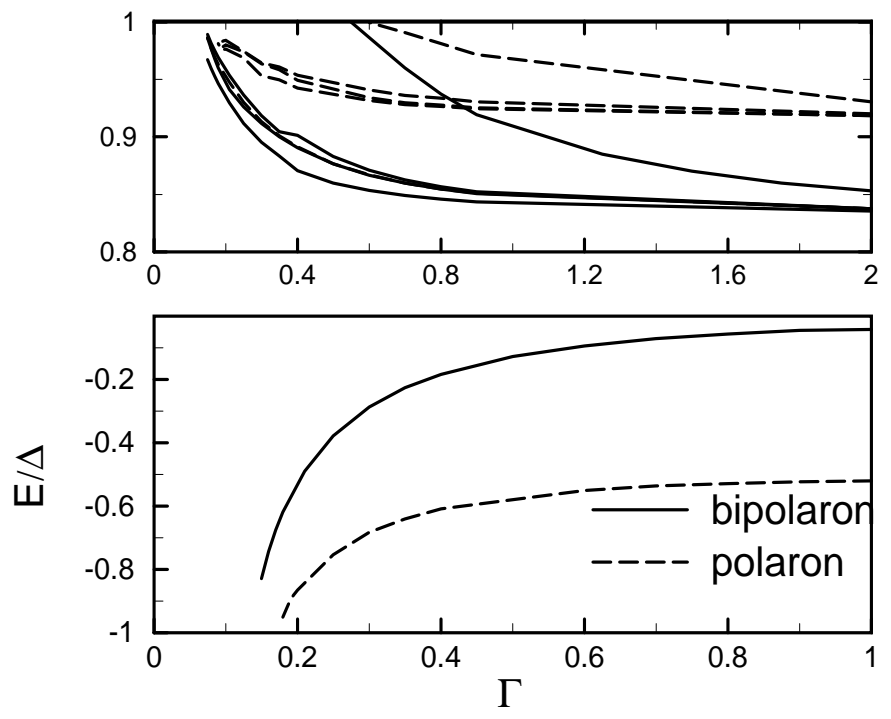

FIG. 7. Energies of localized single particle states lying in the gap. The lower panel shows the states which derive from the valence band; the polaron state is occupied once (spin degeneracy $=2$.) All other states are empty in the ground state. The upper panel, showing states localized below the conduction band edge, has a different scale, both horizontally and vertically.

In principle, these bound states can be seen spectroscopically, as weak mid-gap absorption proportional to doping concentration. We believe that bipolarons are the stable point defects. At $\Gamma=0.3$, the doped-in holes are bound at $\approx 0.7 \Delta$ or $0.7 \mathrm{eV}$ above the top of the lower Peierls band. The optical matrix element for exciting an electron from the valence band into this state (alter- nately, for exciting the mid-gap bound hole to the valence band) is zero in first order at the $\vec{k}=(\pi / 2, \pi / 2, \pi / 2)$ band edge. There is a non-zero matrix element to the $p$ like states split off from the bottom of the upper Peierls band, with energy $\approx 1.88 \Delta$ or $1.88 \mathrm{eV}$. These numbers should not be taken as reliable predictions of actual spectral lines for several reasons. First, the predictions are sure to be somewhat sensitive to the model, especially to the fact that our model omits the disorder potential caused by dopant atoms. Second, there will be significant Franck-Condon effects coming from the fact that if the empty midgap states are suddenly occupied by an optical transition, local oxygen relaxations can lower the energy of these states. Finally, as explained in the companion paper, the Peierls gap of pure $\mathrm{BaBiO}_{3}$ in this model is not "clean." When an electron-hole pair is created in the pure material, self-trapping of the electron and hole should occur, causing absorption in Franck-Condon sidebands below the energy of the nominal Peierls gap $2 \Delta$. This may obscure additional spectral features introduced by bipolarons formed by doping.

\section{MOBILITY OF BIPOLARONS}

At $T=0 \mathrm{~K}$, band formation occurs, in principle, with band width exponentially reduced by Huang-Rhys factors 21,22] $\exp (-S)$ coming from vibrational overlap integrals. Let us make a crude estimate for the polaron. To simplify, we work in the strong coupling limit where the polaron charge is all at one site, $\vec{\ell}$. The state $\mid \overrightarrow{\ell P}>$ representing the polaron at this site, is a product of a hole wavefunction $c_{\ell} \mid$ vac $>$ (where $\mid$ vac $>$ is the electronic vacuum) times a vibrational wavefunction $\mid e_{1}(\vec{\ell})>$ (the vibrational ground state with six oxygens in altered breathing positions around site $\vec{\ell}$.) We want the effective hopping matrix element from this state to a degenerate polaron state $\mid \overrightarrow{\ell^{\prime}} P>$ located at a neighbor site $\overrightarrow{\ell^{\prime}}$. The Hamiltonian matrix element $<\overrightarrow{\ell P}|\mathcal{H}| \overrightarrow{\ell^{\prime}} P>$ factorizes into

$$
t_{\mathrm{eff}}=<\operatorname{vac}\left|c_{\ell}^{\dagger} \mathcal{H} c_{\ell^{\prime}}\right| \operatorname{vac}><e_{1}(\vec{\ell}) \mid e_{1}\left(\overrightarrow{\ell^{\prime}}\right)>
$$

The first factor of Eq. (8) is the matrix element to hop from $\vec{\ell}$ to $\overrightarrow{\ell^{\prime}}$, both being on the A sublattice (since that is where the polarons reside.) This is a secondneighbor hop on the underlying simple cubic lattice. Our Hamiltonian omitted second-neighbor hops, but can now be extended to include such a hopping matrix element $t^{\prime}$, smaller than the first-neighbor hopping $t$. Alternately, we could do second-order degenerate perturbation theory, which would be rather messy and would probably yield something of the same magnitude.

The second factor of Eq. (8) is the "Huang-Rhys factor," namely the overlap between vibrational ground states of two different lattice configurations. Twelve oxygen atoms change locations between the two states. Six 
oxygens around the original polaron site change positions from $u_{1}$ to $u_{0}$, and six oxygens around the new polaron site change positions from $u_{0}$ to $u_{1}$. The corresponding overlap parameter is

$$
\exp (-S)=\exp \left[-12(M \omega / \hbar)\left(u_{0}-u_{1}\right)^{2} / 4\right] .
$$

The exponent, rewritten as $S=(\Delta / \hbar \omega)(\Delta / t)(1 / 48 \Gamma)$, is approximately 2.8 . The polaron band width is thus of order $0.06 \mathrm{eV}$, if we use the band-width $4 \mathrm{eV}$, and assume that the second-neighbor hopping is approximately $t^{\prime}=t / 4$. The disorder caused by the dopant atoms is undoubtedly larger than this, so we should expect polarons to be Anderson-localized.

The bipolaron band-width will be much narrower. For two electrons to hop to second neighbors is a higher order process, and the Huang-Rhys exponent $S$ is larger by 4 since oxygens move the full amount $u_{0}$ rather than by $\left|u_{0}-u_{1}\right|=u_{0} / 2$. Thus the Huang-Rhys factor for bipolarons is smaller by $2 \times 10^{-4}$ than for polarons, and we expect bipolarons to be completely immobile at $T=0$ K.

At high temperature $\left(k_{B} T \approx \hbar \omega\right)$, bipolaron mobility should reduce to the classical version, attempt frequency times thermal activation factor. Assuming that thermal activation is given by $\exp \left(-E_{S} / k_{B} T\right)$, with $E_{S}$ the energy of the saddle point, we can make a plausible argument for how mobility should behave at high $T$. The saddle point is illustrated in Fig. 8. In this state, the bipolaron has split into two polarons located at A-sublattice first neighbors, with full lattice relaxation to minimize the energy at the saddle. This state lies above the bipolaron state in energy by the energy required to unbind a bipolaron into a first-neighbor polaron pair, $\Delta / 2 \approx$ $0.5 \mathrm{eV}$ in strong-coupling approximation. For the actual value $\Gamma=0.3$, we estimate the activation energy to be $E_{S} \approx \Delta / 3 \approx 0.3 \mathrm{eV}$. Experimental measurements 23] vary from $0.17 \mathrm{eV}$ to $0.27 \mathrm{eV}$.
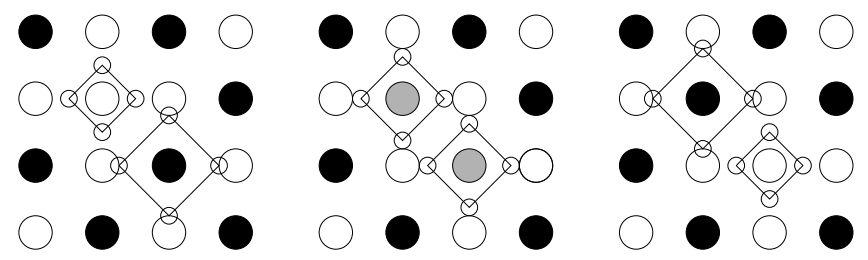

FIG. 8. Hopping of a small bipolaron through an intermediate state with two polarons. Filled circles are $\mathrm{Bi}^{3+}$ ions with two electrons, and open circles are $\mathrm{Bi}^{5+}$ ions with no electrons. The left panel has a hole bipolaron in the small square, which evolves into two hole polarons in the middle panel (shaded circles, medium size squares.) One of the polarons then collapses back to $\mathrm{Bi}^{3+}$ ion (right panel, large square) and the bipolaron has moved to a new location (small square).

\section{ACKNOWLEDGMENTS}

We thank R. Bhargava for writing computer programs, and V. Perebeinos and A. Abanov for help and encouragement. This work was supported by NSF grant no. DMR-0089492.

* Present address: Max-Planck-Institut für Kolloid- und Grenzflächenforschung, D-14424 Potsdam, Germany.

$\dagger$ Present address: AIG Financial Products, Westport, CT 06880 .

[1] D.E. Cox and A.W. Sleight, Solid State Commun. 19, 969 (1976).

[2] G. Vielsack and W. Weber, Phys. Rev. B 54, 6614 (1996).

[3] V. Meregalli and S.Y. Savrasov, Phys. Rev. B 57, 14453 (1998).

[4] F. Marsiglio, J. P. Carbotte, A. Puchkov, and T. Timusk, Phys. Rev. B 53, 9433 (1996); H. J. Kaufmann, O. V. Dolgov, and E. K. H. Salje, Phys. Rev. B 58, 9479 (1998).

[5] T. M. Rice and L. Sneddon, Phys. Rev. Letters 47, 689 (1982); P. Prelovsek, T. M. Rice, and F. C. Zhang, J. Phys. C 20, L229 (1987).

[6] S. Tajima, M. Yoshida, N. Koshizuka, H. Sato, and S. Uchida, Phys. Rev. B 46, 1232 (1992).

[7] P. Piekarz and J. Konior, Physica C 329, 121 (2000).

[8] L. F. Mattheiss and D. R. Hamann, Phys. Rev. B 28, 4227 (1983).

[9] A. I. Liechenstein, I. I. Mazin, C. O. Rodriguez, O. Jepsen, O. K. Andersen, and M. Methfessel, Phys. Rev. B 44, 4442 (1991).

[10] P. Blaha, K. Schwarz, G. Vielsack, and W. Weber, in Electronic Properties of High- $T_{c}$ Superconductors and Related Compounds, edited by H. Kuzmany, M. Mehring, and J. Fink (Springer, Berlin, 1990).

[11] K. Kunc, R. Zeyher, A. I. Liechtenstein, M. Methfessel, and O. K. Andersen, Solid State Commun. 80, 325 (1991).

[12] P. B. Allen and V. N. Kostur, Z. Phys. B 104, 613 (1997).

[13] A. V. Puchkov et al. Phys. Rev. B 54, 6686 (1996); S. H. Blanton et al. Phys. Rev. B 47, 996 (1993); M. A. Karlow et al. Phys. Rev. B 48, 6499 (1993); R. P. S. M. Lobo and F. Gervais, Phys. Rev. B 52, 13294 (1995).

[14] G. Thornton and A. J. Jacobson, Acta. Crystallogr. B 34, 351 (1978); C. Chaillout and A. Santoro, Solid State Commun. 65, 1363 (1988); B. Boyce, F. G. Bridges, T. Claeson, T. H. Geballe, G. G. Li, and A. W. Sleight, Phys. Rev. B 44, 6961 (1991).

[15] J. Yu, X.-Y. Chen, and W. P. Su, Phys. Rev. B 41, 344 (1990).

[16] V. N. Kostur and P. B. Allen, Phys. Rev. B 56, 3105 (1997).

[17] P. B. Allen and I. B. Bischofs, companion paper.

[18] V. Perebeinos and P. B. Allen, Phys. Rev. Letters 85, 5178 (2000). 
[19] I. B. Bischofs, MA thesis, SUNY Stony Brook, December 2000.

[20] W.H. Press, B.P. Flannery, S.A. Teukolsky, and W.T. Vetterling, Numerical Recipes in C, (Cambridge University Press, Cambridge, 1988) p.324.

[21] K. Huang and A. Rhys, Proc. R. Soc (London), Ser. A 204, 406 (1950).

[22] G. D. Mahan, Many-Particle Physics (Plenum, New York, 1981), p.269ff; p. 524ff.

[23] Y. Nagata, A. Mishiro, T. Uchida, M. Ohtsuka, and H. Samata, J. Phys. Chem. Solids 60, 1933 (1999); H. Takagi, S. Uchida, S. Tajima, K. Kitazawa, and S. Tanaka, Proc. 18th Int. Conf. Physics of Semiconductors, 1986, p. 1851; T. Hashimoto, H. Kawazoe, and H. Shimamura, Physica C 223, 131 (1994). 